\title{
Réorganisation des voies visuelles induite par des lésions centrales précoces
}

La combinaison, à un stade précoce du développement, d'une destruction des cibles normales des axones rétiniens et de la suppression des afférences de structures voisines (noyaux auditif ou somatosensoriel du thalamus) provoque l'établissement de projections rétiniennes stables vers ces cibles ectopiques rendues vacantes. Les nouvelles connexions établies sont anatomiquement proches de celles existant normalement et sont fonctionnelles. Ces résultats soulignent le rôle de facteurs épigéniques dans l'établissement des circuits cérébraux.

\section{Christine Métin Douglas O. Frost}

\section{ADRESSES}

C. Métin : chargée de recherche à l'Inserm. Institut des neurosciences, département des neurosciences de la vision, Paris VI, 9, quai Saint-Bernard, bât. C, 6e étage, 75005 Paris, France.

D.O. Frost : associate professor. Kennedy laboratory for developmental neurobiology, department of neurology, Massachussetts General Hospital East, 149 13th Street,

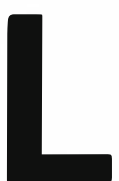

e cerveau des mammiferes se construit grâce à des phénomènes de neurogenèse caractérisés par la surproduction de cellules et de fibres nerveuses, et par des phénomènes régressifs tels que l'élimination des neurones et projections surnuméraires. A partir d'une configuration immature exubérante, ces derniers " sculptent " le système nerveux central (SNC) adulte. Ainsi, parmi les sites de projection des neurones immatures, certains constituent seulement des cibles transitoires. Si la plupart des projections transitoires connectent des structures d'un même système fonctionnel - le système visuel des mammiferes en fournit de fort nombreux exemples, en particulier avec les projections rétinofuges [1-3] et calleuses [4] - certaines connectent des systèmes fonctionnels différents. On connaît, par exemple, des projections transitoires du cortex auditif vers le cortex visuel chez le chaton [5], et des projections transitoires du cortex visuel vers la moelle épinière chez l'embryon de lapin [6] et le rat nouveau-né [7]. Les capacités de réorganisation du SNC des mammiferes à la suite de lésions ou de manipulations expérimentales - chirurgicales ou sensorielles - survenues précocement au cours du développement, sont considérables et font l'objet de nombreuses études. Ces réorganisations peuvent être liées au maintien de projections nerveuses transitoires, ou à des mécanismes responsables de la mise en place de nouvelles voies nerveuses en réponse aux altérations du SNC. La capacité de ces nouveaux réseaux à constituer des circuits fonctionnels compensateurs dépend de leur capacité à reproduire certains aspects du fonctionnement des réseaux nerveux normaux. Les adaptations comportementales dont font généralement preuve les animaux lésés à la naissance, peuvent avoir de 
multiples origines, en particulier l'utilisation accrue de structures intactes, et il n'est pas toujours aisé de les attribuer à des réorganisations anatomiques et fonctionnelles [8].

Notons que le cerveau adulte, incapable des réorganisations observées chez le jeune, reste néanmoins apte à manifester un certain degré de plasticité $[9,10]$.

\section{Réponses du SNC aux lésions et manipulations expérimentales précoces}

Afin de comprendre les mécanismes cellulaires impliqués dans les réponses aux lésions précoces, de nombreux modèles expérimentaux ont été développés chez l'animal. Ils ont permis de démontrer l'importance de deux phénomènes.

L'un de ces phénomènes est la plasticité des processus régressifs et des processus stabilisateurs qui interviennent au cours du développement. Les projections établies transitoirement au cours des phases précoces du développement, projections dites " exubérantes ", sont normalement éliminées par mort cellulaire, perte de collatérales axoniques ou de contacts synaptiques ([11, 12] pour revues). Chez tous les mammiferes où l'expérience a été faite, l'ablation d'un oil à la naissance stabilise la projection rétinofuge ipsilatérale exubérante, normalement transitoire, de l'œil restant [13-15 inter alia]. De même, une énucléation monoculaire ou binoculaire, une privation visuelle monoculaire ou un strabisme conduisent au maintien de projections calleuses visuelles normalement éliminées au cours du développement [16-18]. Toutefois, une privation visuelle binoculaire est sans effet sur la projection calleuse visuelle chez le chat [16], et provoque même une réduction de cette projection par rapport à la normale chez le chat [17]. Ces exemples montrent bien que le contrôle des mécanismes d'élimination/stabilisation est complexe, et influencé à la fois par l'expérience sensorielle et les effets de la dénervation. La part, dans ce contrôle, des facteurs épigénétiques et des facteurs génétiques, n'est pas identique d'une espèce à l'autre [18].

Le second phénomène est la repousse de nouveaux prolongements axonaux $\mathrm{m} / \mathrm{s} n^{\circ} 9$, vol. 6 , novembre 90 par les neurones lésés (sprouting des Anglo-Saxons). Les études réalisées dans diverses espèces montrent qu'au cours des phases précoces du développement, les neurones dont les prolongements axonaux ont été sectionnés peuvent développer de nouvelles collatérales axoniques [19]. Ainsi, Schneider a montré que la destruction du colliculus supérieur chez le hamster nouveau-né provoque un accroissement de la projection rétinienne au noyau latéral postérieur du thalamus [20] ; les fibres rétiniennes lésées développent de nouvelles collatérales vers ce noyau.

Il est intéressant de mentionner que cette propriété semble perdue par les systèmes sensoriels du SNC adulte (voir cependant [9]), vraisemblablement parce que des substances inhibitrices de la croissance des fibres nerveuses sont localisées au niveau de cellules gliales de la matière blanche du SNC adulte [21]. Ces substances inhibitrices sont absentes du système nerveux périphérique ; c'est pourquoi des fragments de nerfs périphériques implantés dans le SNC peuvent jouer le rôle de tunnels dans lesquels des axones centraux sectionnés ont la capacité de repousser [22].

\section{Notre modèle expérimental : induire des projections visuelles stables sur des structures non visuelles chez le hamster}

Il est possible de faire jouer expérimentalement les phénomènes que nous venons de décrire pour créer de nouveaux circuits nerveux. Les rongeurs, et plus particulièrement leur système visuel, sont un matériel de choix pour réaliser ce genre d'expériences. En effet, les rongeurs naissent à un stade de développement précoce avec un cerveau, et en particulier un système visuel, très immature. Ainsi, le hamster naît au $16^{\mathrm{e}}$ jour embryonnaire et ouvre les yeux 15 jours après sa naissance. Pour comparaison, le chat naît au $65^{\mathrm{e}}$ jour embryonnaire et ouvre les yeux 7 jours plus tard, alors que le maca-

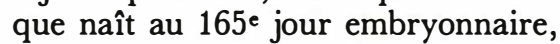
42 jours après que les paupières s'ouvrent, in utero.

Nous avons développé chez le hamster, un modèle expérimental [23] qui s'appuie sur les observations réalisées par Schneider ([20] et voir plus haut). Brièvement, ce dernier avait observé que la destruction du colliculus supérieur chez le hamster nouveau-né, qui, d'une part, prive la rétine de sa principale cible et, d'autre part, " désafférente " partiellement le noyau latéral postérieur, déclenche une repousse des fibres rétiniennes vers ce dernier. Ainsi, des axones rétiniens qui ont perdu leur cible majeure vont occuper un site de projection anormalement vacant. Schneider avait également observé chez les animaux lésés, une repousse des fibres rétiniennes vers le noyau auditif du thalamus.

Dans la filiation de ces travaux nous induisons, au moyen de lésions centrales combinées à des "désafférentations " centrales appropriées, l'établissement de projections rétiniennes stables dans des structures thalamiques non visuelles. Pour cela, les deux cibles principales des axones rétiniens, le colliculus supérieur et le corps genouillé latéral dorsal du thalamus, sont détruites d'un côté du cerveau chez des hamsters nouveaunés. Quand les animaux subissent en même temps, une " désafférentation " du noyau somatosensoriel du thalamus (noyau ventrobasal) ipsilatéral au côté lésé, par transsection des afférences somatosensorielles ascendantes, ils possèdent à l'âge adulte une projection stable de la rétine contralatérale sur le noyau thalamique somatosensoriel (figure $1 A, C$, p. 845). Lorsque la lésion des deux cibles visuelles est associée à une "désafférentation " du noyau auditif du thalamus (noyau genouillé médian) par lésion du colliculus inférieur, les animaux présentent à l'âge adulte une projection stable de la rétine contralatérale sur le noyau auditif du thalamus (figure $1 B, D$ ).

Ces résultats montrent qu'en changeant les facteurs de l'environnement des neurones, on peut agir sur les processus par lesquels ils sélectionnent leurs cibles.

\section{Morphologie \\ des synapses ectopiques en microscopie électronique}

Avant de pouvoir attribuer un rôle fonctionnel réel à ces projections ectopiques, il était nécessaire de montrer que les fibres rétiniennes qui les forment sont capables d'établir des 


\section{RÉFÉRENCES}

1. Rakic P. Prenatal genesis of connections subserving ocular dominance in the rhesus monkey. Nature 1976 ; 261 : 467-71.

2. Land PW, Lund RD. Development of the rat's uncrossed retinotectal pathway and its relation to plasticity studies. Science 1979 ; 205: 698-700.

3. Shatz CJ. The prenatal development of the cat's retinogeniculate pathway. $J$ Neurosci 1983 ; 3 : 482-99.

4. Innocenti GM. Growth and reshaping of axons in the establishment of visual callosal connections. Science $1981 ; 212$ : 824-7.

5. Innocenti GM, Clarke S. Bilateral transitory projection to the visual areas from auditory cortex in kittens. Dev Brain Res $1984 ; 14: 143-8$.

6. Distel H, Hollander H. Autoradiographic tracing of developing subcortical projections of the occipital region in fetal rabbits. J Comp Neurol 1980 ; 192 : 505-18.

7. O'Leary DDM, Stanfield BB. Occipital cortical neurons with transient pyramidal tract axons extend and maintain collaterals to subcortical but not intracortical targets. Brain Res 1985 ; 336 : 326-33.

8. Kolb B, Whishaw IQ. Plasticity in the neocortex : mechanisms underlying recovery from early brain damage. Prog Neurobiol 1989 ; 32 : 235-76.

9. Raisman G. Neuronal plasticity in the septal nuclei of the adult rat. Brain Res 1969 ; 14 : 25-48.

10. Irle E. Lesion size and recovery of function : some new perspectives. Brain Res Review 1987 ; 12 : 307-20.

11. Cowan WM, Fawcett JW, O'Leary DDM, Stanfield BB. Regressive events in neurogenesis. Science 1984; 225 : 1258-65.

12. Finlay BL, Wikler KC, Sengelaub DR. Regressive events in brain development and scenarios for vertebrate brain evolution. 844 relations synaptiques avec les neurones relais des cibles thalamiques non visuelles. Des études neuroanatomiques ont montré que dans les noyaux thalamiques somesthésiques et auditifs des hamsters opérés, les terminaisons rétiniennes sont engagées dans des glomérules synaptiques et établissent des synapses avec les cellules relais [24]. Rappelons que les " glomérules " sont des complexes synaptiques rassemblant divers éléments nerveux - dendrites, terminaisons axonales - qui établissent entre eux de nombreux contacts synaptiques et qui sont séparés du reste du neuropile* par des feuillets cytoplasmiques d'origine astrocytaire. Par ailleurs, cette étude a permis de distinguer l'influence respective de l'afférence et de la cible sur la morphologie des glomérules synaptiques. Pour cela, la morphologie des glomérules dans lesquels sont engagées les terminaisons rétiniennes ectopiques a été analysée en microscopie électronique dans le thalamus somatosensoriel et auditif des animaux opérés. Puis, elle a été comparée à celle des glomérules contenant les terminaisons rétiniennes, somatosensorielles et auditives dans les noyaux thalamiques respectivement visuels, somatosensoriels et auditifs, des animaux normaux [24].

Chez le hamster adulte normal, l'aspect des terminaisons rétiniennes et des glomérules synaptiques dans le corps genouillé latéral dorsal est sensiblement différent de celui des terminaisons sensorielles et des glomérules synaptiques dans les noyaux thalamiques somatosensoriel et auditif. Ces différences incluent la position des glomérules synaptiques sur les neurones cibles, la nature, la taille et le nombre de leurs constituants ainsi que les relations synaptiques, le nombre de synapses établies par chaque élément et la morphologie des mitochondries dans les boutons terminaux sensoriels (figure $2 A, B, D, E$, p. 846).

Chez les hamsters adultes opérés à la naissance, les terminaisons rétiniennes rencontrées dans les noyaux thalamiques somatosensoriels et auditifs,

\footnotetext{
- Neuropile: zone de la substance grise, entre les corps cellulaires et les capillaires sanguins, contenant les synapses dans un enchevêtrement d'axones, de prolongements gliaux et de dendrites.
}

et leurs glomérules synaptiques, ressemblent aux terminaisons et glomérules somatosensoriel et auditif observés chez les animaux normaux. Toutefois, les afférences rétiniennes conservent la structure caractéristique de leurs mitochondries (figure $2 C, F$ ).

Il semble'donc que la différenciation de la plupart des caractéristiques morphologiques des terminaisons axonales et de leurs glomérules synaptiques - à l'exception des mitochondries - soit sous le contrôle des neurones cibles ou de leur environnement. Pour les structures qui réalisent les contacts synaptiques entre neurones, ce contrôle pourrait s'exercer via des interactions entre éléments pré- et postsynaptiques. En revanche, les éléments non impliqués directement dans ces contacts, tels que les organites cellulaires, resteraient indépendants de ce contrôle.

\section{Rétinotopie des connexions visuelles induites chirurgicalement}

L'analyse, par des techniques neuroanatomiques, de la topographie des projections visuelles aberrantes vers le thalamus somatosensoriel et auditif, a montré qu'elles sont organisées de manière rétinotopique, même si la précision de cette rétinotopie est réduite par rapport à celle de la projection visuelle thalamique normale [23]. Chez le hamster normal, comme chez les autres rongeurs, la surface latérale du corps genouillé latéral dorsal contient une représentation topographique de la rétine contralatérale. L'axe inféro-supérieur de la rétine est représenté suivant l'axe rostro-caudal du noyau, alors que l'axe naso-temporal de la rétine est représenté suivant l'axe ventrodorsal du noyau. Dans l'épaisseur du corps genouillé latéral dorsal, chaque point de la rétine se projette sur un cylindre de tissu incliné suivant un axe caudo-latéral/rostro-médian. Dans les deux groupes d'animaux opérés, le même pôle rétinien est toujours représenté au même endroit de la projection rétinienne ectopique et, d'un animal à l'autre, les représentations des différents pôles rétiniens conservent la même position relative dans les cibles ectopiques.

Des fibres rétiniennes sont donc capables d'établir une projection 
ordonnée dans un noyau thalamique non visuel, dont elles sont normalement absentes. Il faut toutefois noter que les fibres qui se terminent dans le thalamus somatosensoriel traversent le corps genouillé résiduel, ce qui leur donne la possibilité, à un moment de leur développement, d'interagir avec leurs cellules cibles normales. Les fibres qui se terminent dans le thalamus auditif n'ont pas cette possibilité.

Ces résultats peuvent aider à la compréhension des mécanismes impliqués dans la mise en place de cartes topographiques dans le cerveau. Deux groupes d'hypothèses relatives au guidage axonal des fibres qui participent à une projection topographiquement ordonnée (pour revue [25]) peuvent rendre compte des résultats obtenus chez les animaux opérés : (1) l'ordre des terminaisons dans la cible dépendrait fortement de l'agencement des fibres dans la voie afférente, le gradient temporel de croissance des fibres et/ou les interactions fibre/fibre jouant un rôle important ; (2) suivant le modèle de chémoaffinité défini par Sperry [26], des fac-

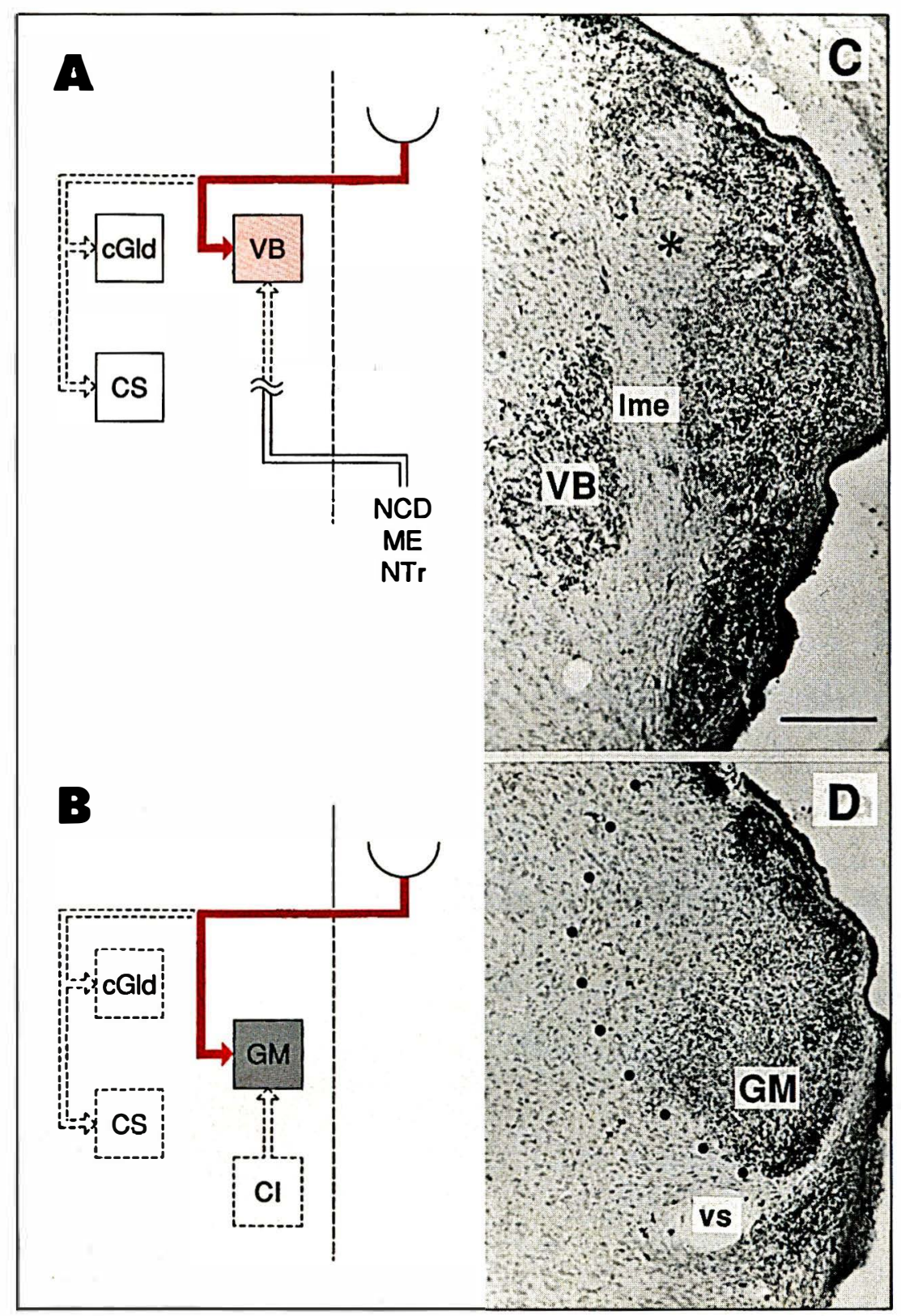

$m / s n^{\circ} 9$, vol. 6, novembre 90
Figure 1. Procédure expérimentale : induction chirurgicale de projections visuelles ectopiques. A et $B$. Les structures représentées en pointillés sont détruites à la naissance. Les voies aberrantes résultant de cette chirurgie sont indiquées en trait plein ; elles forment chez l'adulte des connexions stables entre la rétine et le noyau somatosensoriel du thalamus (noyau ventrobasal, VB, (A) ou entre la rétine et le noyau auditif du thalamus Inoyau genouillé médian, GM, (B).cGld : corps genouillé latéral dorsal, éliminé par dégénérescence rétrograde après cautérisation du cortex occipital. $\mathrm{CS}$ et $\mathrm{Cl}$ : colliculus supérieur et colliculus inférieur, détruits par cautérisation. NCD : noyaux des colonnes dorsales; ME : moelle épinière; NTr: noyau trijumeau. La transsection des voies somatosensorielles ascendantes est effectuée au niveau du colliculus supérieur (tiers antérieur). La ligne brisée indique le plan de symétrie bilatérale de l'animal. $\boldsymbol{C}$ et $\boldsymbol{D}$. Projections rétiniennes aberrantes au VB (C) et au GM (D) marquées de façon antérograde avec la peroxydase du raifort (HRP) chez de jeunes hamsters adultes 111 semaines). Coupes frontales du thalamus ipsilatéral aux structures lésées à la naissance, et controlatéral à l'œil injecté de HRP. HRP révélée par la tétraméthylbenzidine (TMB) sur des coupes de $40 \mu \mathrm{m}$ d'épaisseur, colorées au rouge neutre. Remarquer, en C, qu'une lésion incomplète du cortex visuel a épargné une partie du cGld; la région marquée * correspond au site de terminaison dans le cGld des afférences rétiniennes issues de l'œil ipsilatéral, non colorées par la HRP. vs: vaisseau sanguin; Ime : lame médullaire externe. Échelle : $180 \mu \mathrm{m}$. 


\section{RÉFÉRENCES}

13. Rakic P, Riley KP. Overproduction and elimination of retinal axons in the fetal rhesus monkey. Science $1983 ; 219$ : 1441-4.

14. Williams RW, Bastiani MJ, Chalupa LM. Loss of axons from the cat optic nerve following fetal unilateral enucleation : an electron microscopic analysis. $J$ Neurosci $1983 ; 1$ : 133-44.

15. Godement $P$, Salaün J, Métin C. Fate of uncrossed retinal projections following early or late prenatal monocular enucleation in the mouse. J Comp Neurol $1987 ; 255$ : 97-109.

16. Cusick CG, Lund RD. Modification of visual projections in rats. J Comp Neurol 1982 ; 212 : 385-98.

17. Innocenti GM, Frost DO. The postnatal development of visual callosal connections in the absence of visual experience or of the eyes. Exp Brain Res 1980; 39 : 365-75.

18. Dehay C, Horsburgh G, Berland M, Killackey $\mathrm{H}$, Kennedy $\mathrm{H}$. Maturation and connectivity of the visual cortex in monkey is altered by prenatal removal of retina input. Nature 1989 ; 337 : 265-7.

19. Kalil K, Reh T. Regrowth of severed axons in the neonatal central nervous system : establishment- of normal connections. Science 1979 ; 1158-60.

20. Schneider GE. Early lesions of the superior colliculus : factors affecting the for-. mation of abnormal retinal projections. Brain Behav Evol 1973 ; 8 : 73-109.

21. Savio T, Schwab ME. Rat CNS white matter, but not gray matter, is nonpermissive for neuronal cell adhesion and fiber outgrowth. J Neurosci 1989 ; 9 (4) : 1126-33.

22. Aguayo AJ. Axonal regeneration from injured neurons in the adult mammalian central nervous system. In : Cotman CW, ed. Synaptic Plasticity and Remodeling. New York: Guilford Press, 1985 : 457-83.

23. Frost DO. Orderly anomalous retinal projections to the medial geniculate, ventrobasal and lateral posterior nuclei of the hamster. J Comp Neurol 1981 ; 203 : 227-56.

24. Campbell G, Frost DO. Synaptic organization of anomalous retinal projections to somatosensory and auditory thalamus : target-controled morphogenesis of axon terminals and synaptic glomeruli. J Comp Neurol $1988 ; 272: 383-408$. teurs chimiques distribués en gradient dans le cerveau seraient capables d'interagir spécifiquement avec certaines catégories de fibres en croissance et d'orienter leur croissance vers un site particulier de la cible. Ces facteurs qui, dans les conditions normales, agiraient sur l'afférence sensorielle spécifique, seraient également capables d'interagir avec une afférence sensorielle ectopique. Ces deux hypothèses sont généralement associées dans les modèles théoriques qui cherchent à rendre compte de la formation des cartes dans le cerveau [27]. Mais la topographie finale résulte probablement d'une coopération - simultanée ou décalée dans le temps - entre des mécanismes de guidage axonal et des mécanismes dépendants de l'activité électrique des fibres nerveuses (voir plus loin).

\section{Capacité fonctionnelle} des voies visuelles ectopiques

Les études neuroanatomiques montrent que les projections visuelles ectopiques sont organisées de manière rétinotopique et qu'elles établissent dans les cibles non visuelles, des synapses au sein de glomérules synaptiques. L'information visuelle transmise par une voie rétinienne ectopique peut-elle être traitée en tant que telle par les neurones relais du

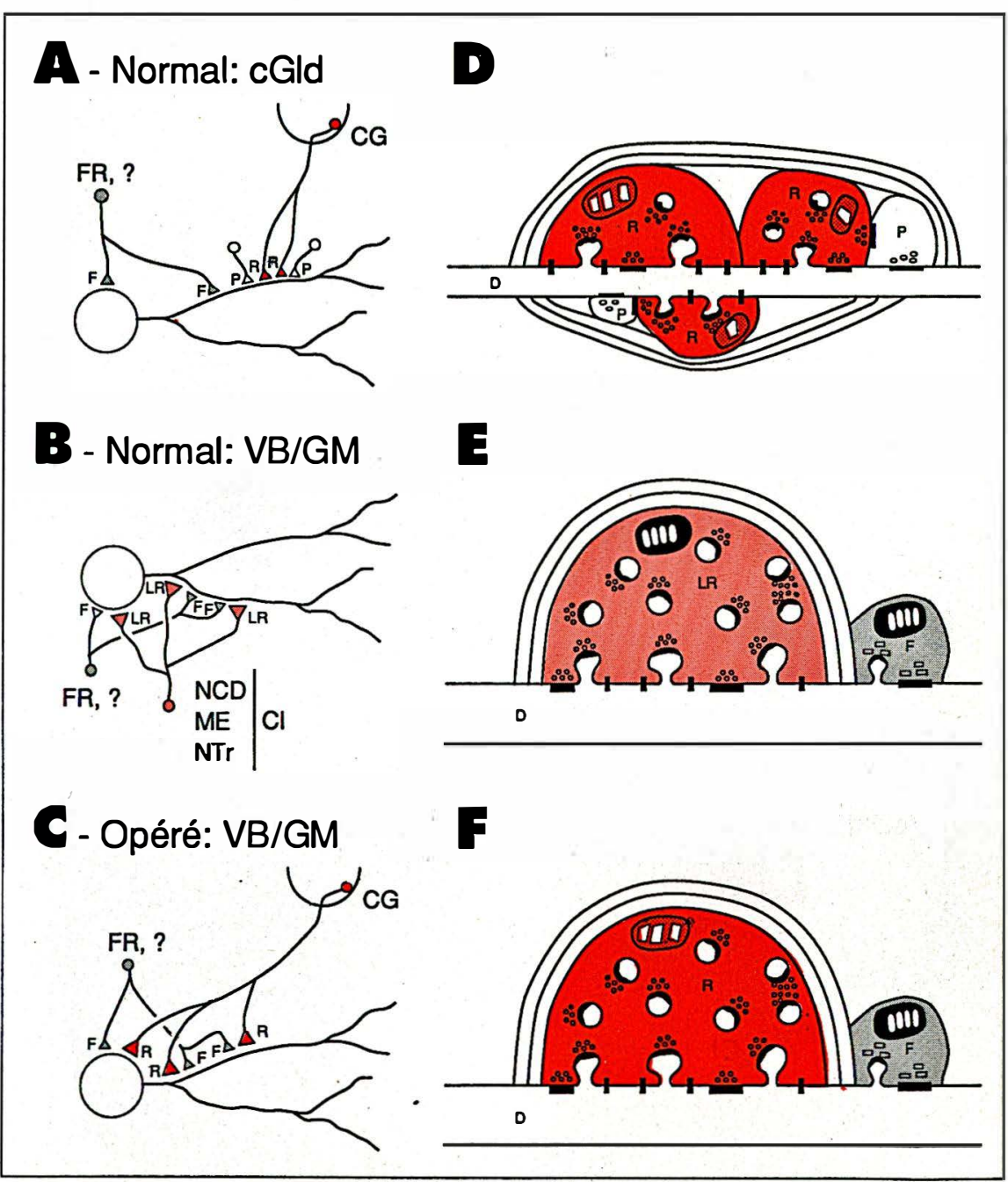


thalamus, puis par ceux de la cible corticale des neurones thalamiques? Et dans l'affirmative, de quelle manière ? Pour répondre à ces questions, nous avons entrepris l'étude fonctionnelle de la voie visuelle rétine - thalamus somatosensoriel cortex somatosensoriel, au moyen d'enregistrements unitaires et multiunitaires du cortex somatosensoriel (aires S1 et S2) de hamsters possédant cette voie aberrante. Des enregistrements similaires ont été menés en parallèle dans le cortex visuel de hamsters normaux.

Les enregistrements multi-unitaires [28] ont révélé qu'il est possible de recueillir des réponses à une stimu- lation visuelle dans les aires $\mathrm{S} 1$ et $\mathrm{S} 2$ du cortex somatosensoriel d'animaux opérés, alors que ce n'est jamais le cas chez l'animal normal. En outre, ces deux aires contiennent chacune une représentation topographique de l'axe inféro-supérieur du champ visuel, les deux représentations faisant un angle de 90 degrés entre elles. Or les représentations somatotopiques contenues en S1 et S2 chez ces animaux, comme chez les normaux, font aussi entre elles un angle de 90 degrés. En revanche, il n'y a pas de représentation ordonnée de l'axe naso-temporal. Cette topographie est cohérente avec la manière dont la projection thalamo-corticale somatosensorielle intercepte la projection visuelle ectopique dans le thalamus somatosensoriel [28]. Ces résultats laissent penser que les synapses formées par les axones rétiniens sur les cellules relais du thalamus somatosensoriel sont fonctionnelles. Il faut noter qu'au cours des enregistrements effectués dans le cortex somatosensoriel, nous avons recueilli des réponses visuelles, des réponses somatosensorielles et parfois des réponses bimodales. Or, la transsection de l'afférence somatosensorielle ascendante est effectuée chez les animaux nouveau-nés à un moment où cette voie est en plein développement. Elle ré-innerve donc sa cible

Figure 2. Organisation synaptique des projections rétiniennes ectopiques. A, B, C : constituants des glomérules synaptiques et localisation sur les neurones cibles. D, E, F : schémas résumant la morphologie des constituants glomérulaires et leurs relations synaptiques telles qu'elles apparaissent en microscopie électronique. $\mathbf{A}$ et $\mathbf{D}$ : corps genouillé latéral dorsal (cGld) normal. A : les boutons $R$ des terminaisons axonales des cellules ganglionnaires de la rétine (CG) participent aux glomérules localisés sur les régions intermédiaires/proximales des dendrites des neurones relais du cGld. Boutons $P$ des dendrites présynaptiques d'interneurones du cGld et boutons $F$ (peu fréquents) dont certains proviennent $d^{\prime}$ axones réticulo-thalamiques (FR: formation réticulée). D: organisation synaptique des glomérules. Les boutons $R$ (plusieurs par glomérule) établissent des synapses asymétriques avec le tronc ou les épines dendritiques des cellules relais. IIs établissent aussi des contacts synaptiques avec les boutons $P$, eux-mêmes présynaptiques aux dendrites des neurones relais. Les boutons $F$ (non illustrés) sont soit à l'intérieur du glomérule, soit à l'extérieur contre la capsule gliale et font des synapses symétriques avec les dendrites des neurones relais. Les boutons $R$, de taille petite ou moyenne, ont des mitochondries claires avec des crêtes dilatées et irrégulières. B et $\mathbf{E}$ : noyau ventrobasal (VB) et genouillé médian (GM) normal. B : les boutons LR des axones somatosensoriels ou des axones auditifs ascendants participent aux glomérules situés sur les régions proximales des dendrites des neurones relais du VB et du GM, ou sont associés à leurs corps cellulaires. Les boutons $P$ sont très rares, les boutons $F$ très fréquents. NCD, ME, $\mathrm{NTr}, \mathrm{Cl}$ : voir légende figure 1. E : organisation des glomérules. Un seul bouton LR par glomérule, le plus souvent, établit des synapses asymétriques avec le tronc, les épines dendritiques ou somatiques, ou le soma des neurones relais. Des boutons $F$ sont presque toujours associés aux glomérules, à l'extérieur de la capsule gliale, et établissent des synapses symétriques sur les dendrites ou les corps cellulaires des cellules relais. Les boutons LR sont moyens à très larges et possèdent des mitochondries sombres avec des crêtes fines et régulières. $\boldsymbol{C}$ et $\boldsymbol{F}$ : noyau ventrobasal $(V B)$ et genouillé médian (GM) des animaux opérés. $C$ : les boutons $R$ des cellules ganglionnaires rétiniennes remplacent les boutons $L R$ des afférences spécifiques. Les glomérules occupent la même position que dans le VB et le GM normal et les autres boutons sont ceux observés dans le VB et le GM normal. F : organisation des glomérules dans le site de terminaison des afférences rétiniennes. Chaque glomérule contient un seul bouton $R$ qui établit des synapses asymétriques avec le tronc, les épines dendritiques ou somatiques ou le soma des neurones relais. Les boutons $P$ sont très rares. Des boutons $F$ sont presque toujours associés aux glomérules, à l'extérieur de la capsule gliale, et établissent des synapses symétriques sur les dendrites ou les corps cellulaires des cellules relais. Les boutons $R$ sont moyens à très larges et possèdent des mitochondries claires avec des crêtes dilatées et irrégulières. Ces glomérules possèdent les caractères morphologiques des glomérules normalement rencontrés dans le VB et le GM, à l'exception des mitochondries. 


\section{RÉFÉRENCES}

25. Udin SB, Fawcett JW. Formation of topographic maps. Ann Rev Neurosci 1988 ; 11 : 289-327.

26. Sperry RW. Chemoaffinity in the orderly growth of nerve fiber pattern and connections. Proc Natl Acad Si USA 1963 ; $50: 703-10$

27. Fraser SE, Hunt RK. Retinotectal specificity : models and experiments in search of a mapping function. Ann Rev Neurosci $1980 ; 3: 319-52$.

28. Frost DO, Métin C. Induction of functional retinal projections to the somatosensory system. Nature $1985 ; 317$ : 162-4.

29. Métin C, Frost DO. Visual responses of neurons in somatosensory cortex of hamsters with experimentally induced retinal projections to somatosensory thalamus. Proc Natl Acad Si USA 1989 ; 86 : 357-61.

30. Sur M, Garraghty PE, Roe AW. Experimentally induced visual projections into auditory thalamus and cortex. Science 1988 ; 242 : 1437-41.

31. Frost DO. Axonal growth and target selection during development : retinal projections to the ventrobasal complex and other "non-visual " structures in neonatal Syrian hamsters. J Comp Neurol 1984; 230 : 576-92.

32. Jhavery S, Edwards MA, Schneider GE. Two stages of growth during development of hamster's optic tract. Anat Rec $1983 ; 205: 225$.

33. Bhide P, Frost DO. Development of optic tract axons in the hamster. 1990; soumis.

34. Innocenti GM, Clarke S, Kraftsik R. Interchange of callosal and association projections in the developing visual cortex. $J$ Neurosi $1986 ; 6(5)$ : 1384-409.

35. O'Leary DDM, Stanfield BB. Selective elimination of axons extended by developing cortical neurons is dependent on regional locale : experiments utilizing fetal cortical transplants. J Neurosci $1989 ; 9(7): 2230-46$.

36. Bhide PG, West WC, Frost DO. Development of permanent retinal projection to somatosensory and auditory thalamic nuclei in hamster : a Dil study. Neurosci Soc Abstr
Figure 3. Champs récepteurs visuels et propriétés fonctionnelles des neurones enregistrés dans le cortex visuel (aire $V 1$ ) de hamsters normaux et dans le cortex somatosensoriel (aires S1, S2) de hamsters opérés à la naissance. Champs récepteurs : organisation spatiale et localisation dans le champ visuel des champs récepteurs de neurones visuels enregistrés, à gauche, dans l'aire visuelle primaire de hamsters normaux $(1 a, 2 a, 3 a)$, et à droite, dans les aires somatosensorielles de hamsters possédant la voie visuelle aberrante rétine-thalamus somatosensoriel $(1 b, 2 b, 3 b)$. Les limites des champs récepteurs et la distribution des zones de réponse à l'apparition d'un stimulus lumineux (zones ON " + ), à la disparition d'un stimulus lumineux (zones OFF " - "), ou aux deux (zones ON-OFF " \pm ") ont été déterminées à la main. La représentation sphérique du champ visuel est partielle. Le méridien vertical 0 correspond à la projection du plan de symétrie de l'animal dans le champ visuel, et le méridien horizontal $O$ à celle du plan horizontal contenant ses yeux. Lignes d'isoazimut et d'isoélévation espacées de 30 degrés. Étoile encerclée : projection du disque optique de la rétine. Réponses, présentées sous forme de diagramme polaire, des mêmes neurones à un stimulus visuel. Les neurones $1 a$ et 16 ont des champs récepteurs (CR) sélectifs à l'orientation : ils répondent préférentiellement à une barre lumineuse, présentée dans leur CR suivant une orientation particulière. Les diagrammes $1 \mathrm{a}$ et $1 \mathrm{~b}$ montrent la réponse de ces neurones à une barre stationnaire, présentée dans leur $C R$ suivant les orientations matérialisées par les rayons des diagrammes. Les 6 orientations testées (O est vertical, 90 est horizontal) sont réparties dans une séquence de stimulation aléatoire répétée au moins 5 fois. La longueur de chaque rayon pointillé est proportionnelle à la réponse moyenne du neurone, quand on stimule son CR avec une barre orientée comme ce rayon. L'écart type de la réponse est proportionnel au segment qui prolonge le rayon. Si la barre se déplace, la direction de mouvement préférée est orthogonale à l'orientation optimale (non illustré). Les neurones $2 a$ et $2 b$ ont des CR non orientés sélectifs à la direction : ils n'ont aucune préférence pour la forme (tache ou barre) et l'orientation du stimulus, mais répondent préférentiellement lorsque le stimulus se déplace suivant une direction particulière. Les diagrammes $2 a$ et $2 b$ montrent la réponse de ces neurones à un stimulus en mouvement, se déplaçant à travers leur CR suivant les différentes directions matérialisées par les rayons des diagrammes. Les 12 directions testées 10 est un mouvement naso $\rightarrow$ temporal dans le champ visuel, 90 un mouvement supero $\rightarrow$ inférieur) surviennent dans une séquence de stimulation aléatoire répétée au moins 5 fois. La longueur de chaque rayon pointillé est proportionnelle à la réponse évoquée moyenne du neurone, quand on stimule son CR suivant la direction matérialisée par ce rayon; le segment qui le prolonge est proportionnel à l'écart type de la réponse. Les neurones $3 a$ et $3 b$ ont des $C R$ non orientés et non directionnels : ils n'ont de préférence ni pour la forme et l'orientation du stimulus, ni pour sa direction de mouvement. Les diagrammes polaires, construits comme les diagrammes $2 a$ et $2 b$, illustrent ce dernier point. normale dans la suite du développement postnatal, ce qui conduit à une double innervation du noyau thalamique chez l'animal adulte : visuelle d'une part, somatosensorielle d'autre part. A la lumière des résultats électrophysiologiques, il semble que la région innervée par les afférences visuelles le soit également par les afférences somesthésiques. Ces résultats doivent être précisés par une analyse détaillée des interactions entre les deux modalités sensorielles, analyse actuellement en cours.

Des enregistrements unitaires [29] ont mis en évidence que les neurones visuels enregistrés dans le cortex somatosensoriel des animaux opérés à la naissance ont des propriétés fonctionnelles qui ressemblent à celles des neurones visuels enregistrés dans le cortex visuel primaire de hamsters adultes normaux (figure 3).

En particulier, dans les deux cas, les neurones possèdent des champs récepteurs bien définis avec des zones qui répondent à la présentation d'un stimulus lumineux (zones $\mathrm{ON}$ ), ou à l'extinction d'un stimulus lumineux (zones OFF), ou aux deux (zones 


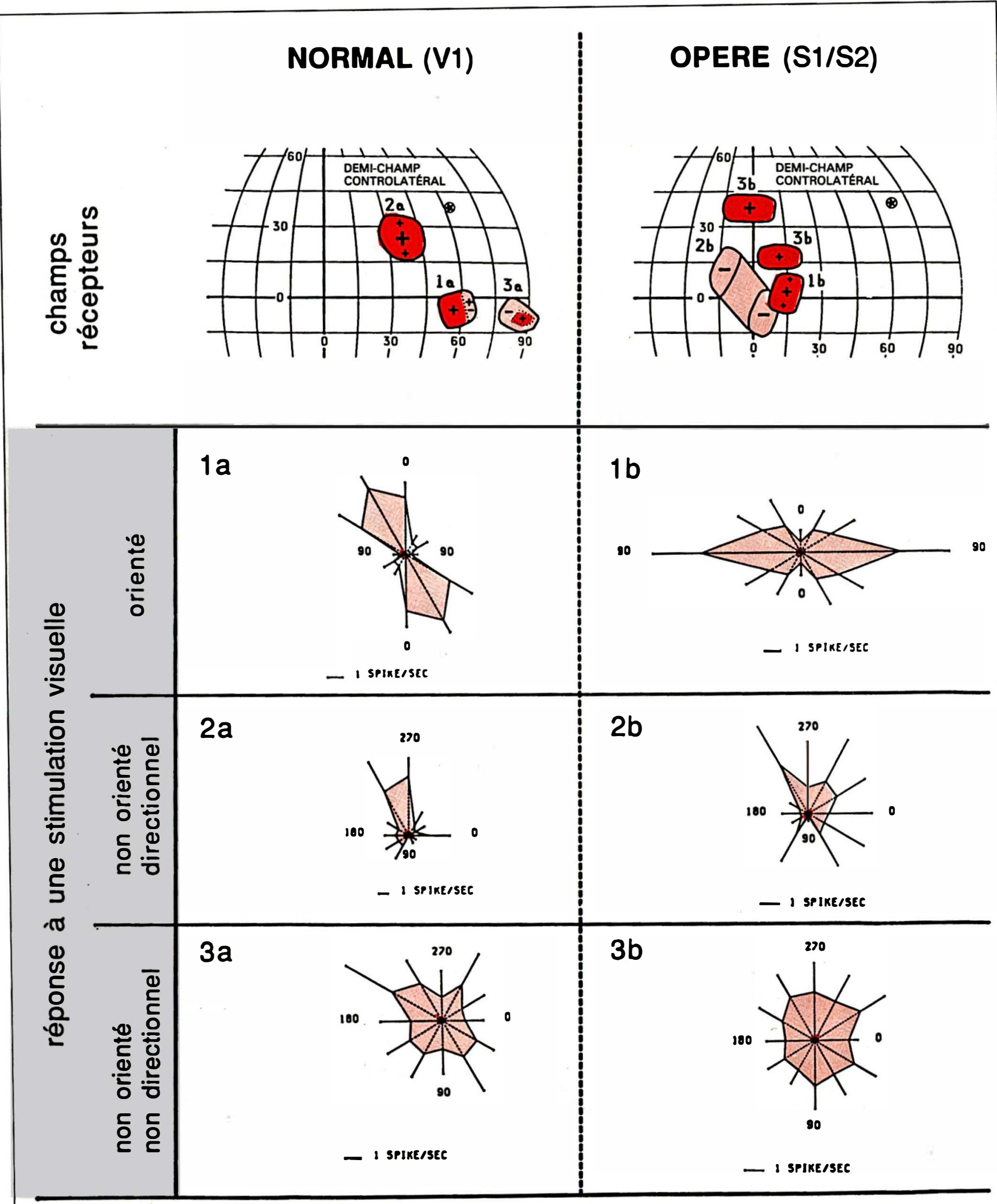

$\mathrm{m} / \mathrm{s} n^{\circ} 9$, ool. 6 , novembre 90 


\section{RÉFÉRENCES}

37. Frost DO. Development of surgically induced retinal projections to the medial geniculate, ventrobasal and lateral posterior nuclei in Syrian hamsters : a quantitative study. J Comp Neurol 1986 ; 252 : 95-105.

38. Walicke PA. Novel neurotrophic factors, receptors, and oncogenes. Ann Rev Neurosci $1989 ; 12$ : 103-26.

39. Archer SM, Dubin MW, Stark LA. Abnormal development of kitten retinogeniculate connectivity in the absence of action potentials. Science 1982 ; 217 : 743-5.

40. Stryker MP, Harris WA. Binocular impulse blockade prevents the formation of ocular dominance columns in cat visual cortex. $J$ Neurosci $1986 ; 6: 2117-33$.

41. Stent GS. A physiological mechanism for Hebb's postulate of learning. Proc Natl Acad Sci USA 1973 ; 70 : 997-1001.

42. Changeux JP, Danchin A. Selective stabilization of developing synapses as a mechanism for the specification of neuronal networks. Nature 1976 ; 264 : 705-12.

43. Freeman JM, Frost DO. Synapse formation by optic tract axons that project transiently to somatosensory and auditory nuclei in the neonatal hamster. Neurosci Abstr 1987 ; 13 : 1023.

44. Shatz CJ, Kirkwood PA. Prenatal development of functional connections in the cat's retinogeniculate pathway. $J$ Neurosci $1984 ; 4$ : 1378-97.

45. Sherman SM, Spear PD. Organization of visual pathways in normal and visually deprived cats. Physiol Rev $1982 ; 62$ : 738-855.

46. Schneider GE. Mechanisms of functional recovery following lesions of visual cortex or superior colliculus in neonate and adult hamsters. Brain Behav Evol 1970 ; 3 : 295-323.

47. Rudel RG, Teuber H-L. Spatial orientation in normal children and in children with early brain injury. Neuropsychologia $1971 ; 9: 401-7$.
ON-OFF). Certains sont composés d'une zone de réponse, d'autres de plusieurs zones concentriques ou adjacentes. De plus, les mêmes catégories fonctionnelles de neurones sont rencontrées dans les deux groupes expérimentaux, à savoir des neurones sélectifs à l'orientation et à la direction du mouvement, des neurones non sélectifs à l'orientation mais sélectifs à la direction du mouvement, et enfin des neurones qui ne sont sélectifs ni à l'orientation ni à la direction. Enfin, dans les deux cas, la distribution laminaire de ces types fonctionnels de neurones montre une prépondérance de neurones sélectifs à l'orientation par rapport aux autres types fonctionnels dans les couches supragranulaires.

Toutefois, il existe certaines différences entre les propriétés fonctionnelles des neurones visuels enregistrés dans le cortex visuel des animaux normaux et dans le cortex somatosensoriel des animaux opérés. Alors que les champs récepteurs des neurones de l'aire visuelle primaire recouvrent uniformément une région du champ visuel d'environ 90 degrés ${ }^{2}$, les champs récepteurs des neurones visuels du cortex somatosensoriel comprennent fréquemment deux zones de réponse stable et facilement caractérisable, disjointes dans le champ visuel. Elles couvrent en moyenne 275 degrés $^{2}$ et sont reliées entre elles par une région où la réponse évoquée visuelle est plus irrégulière et difficile à caractériser. De plus, les neurones visuels enregistrés dans le cortex somatosensoriel préfèrent généralement des vitesses de mouvement plus rapides que les neurones de l'aire visuelle primaire ; leur activité spontanée ainsi que la variabilité de leurs réponses évoquées sont significativement plus élevées que celle des neurones de l'aire visuelle primaire. Enfin, environ le quart des neurones visuels enregistrés dans le cortex somatosensoriel répondent également à une stimulation somatosensorielle, avec un champ récepteur localisé au niveau de la tête.

Ces résultats électrophysiologiques permettent, en outre, d'avancer deux types d'hypothèses, non nécessairement exclusives, quant au traitement de l'information par les systèmes sen- soriels : 1) dans les voies visuelles et somatosensorielles, les noyaux thalamiques et les aires corticales effectueraient, à des niveaux correspondants, le même type de transformation sur l'information sensorielle afférente ; le système somatosensoriel serait donc capable de traiter une information visuelle, pour autant qu'il la reçoive ; 2) la capacité d'une voie sensorielle à traiter une information d'un type donné serait en partie déterminée par l'organisation du message afférent qu'elle reçoit au cours de son développement. Ainsi, la voie somatosensorielle des hamsters opérés, qui a reçu précocement au cours de son développement des informations visuelles et somesthésiques, serait devenue une voie bimodale.

Par une chirurgie comparable à celle développée chez le hamster, il est possible d'induire chez un carnivore, le furet, le développement de projections visuelles ectopiques vers le système auditif [30]. Des réponses visuelles ont été enregistrées dans le cortex auditif de furets opérés, mais les cellules semblent donner de moins bonnes réponses que celles que nous avons recueillies dans le cortex somatosensoriel du hamster. Elles semblent également moins sélectives aux différents paramètres de la stimulation visuelle. Indépendamment de variations interspécifiques, ces différences peuvent provenir de ce que la chirurgie est effectuée plus tard chez le furet que chez le hamster, et affecte la projection rétinofuge à un stade différent du développement.

\section{Développement normal de projections visuelles chez le hamster}

Rappelons que deux processus pouvant rendre compte des réorganisations induites dans le SNC immature par des lésions sont, d'une part, la plasticité des processus de régression et de stabilisation et, d'autre part, la repousse de collatérales axoniques. Le premier de ces processus ne peut s'exercer que sur un ensemble de connexions immature et exubérant. Or, la rétine du hamster nouveau-né se projette non seulement vers les cibles visuelles thalamiques et mésencéphaliques, mais aussi vers le noyau somatosensoriel - ventrobasal - du 


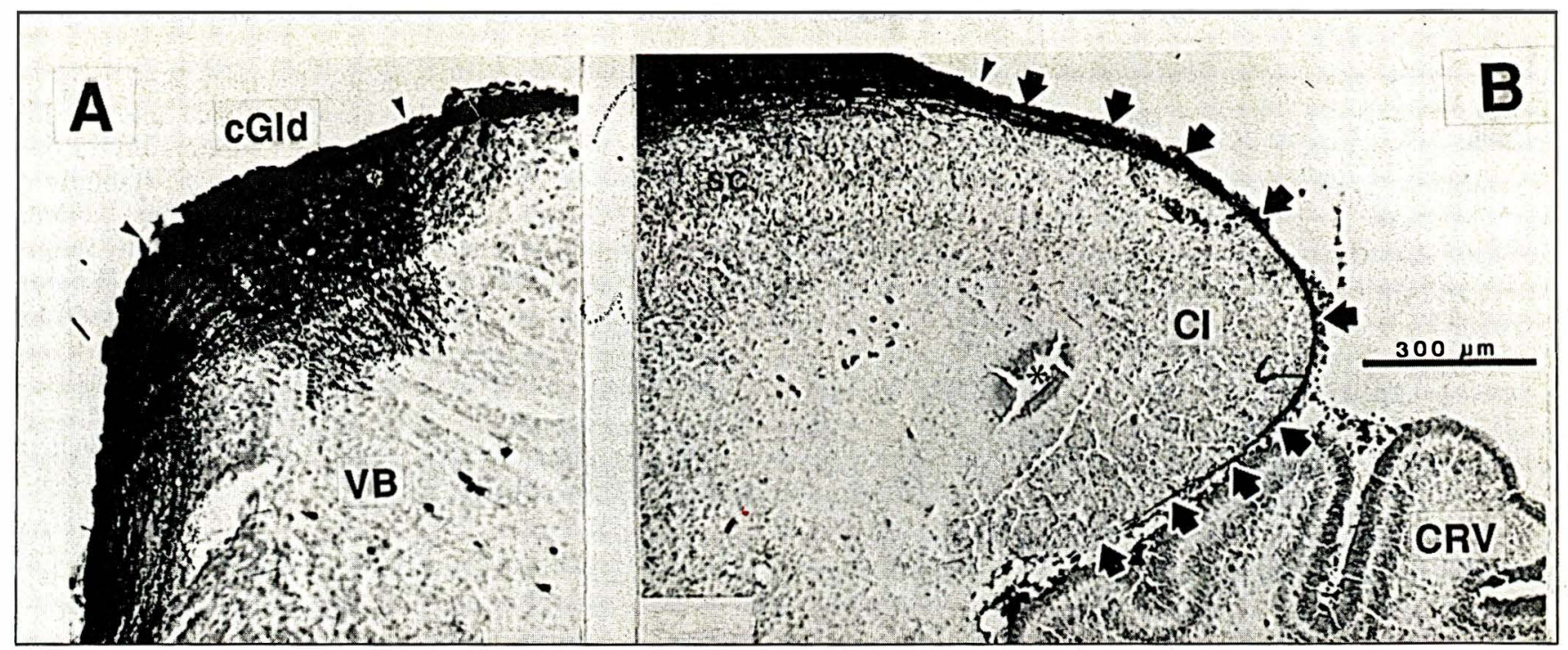

Figure 4. Projections rétiniennes transitoires chez le hamster normal. A. Marquage antérograde de la projection rétino-VB transitoire chez un hamster de 2 jours, après injection de peroxydase HRP (horse-radish peroxydase) dans l'œil contralatéral. Coupe frontale, face dorsale en haut, bord médian à droite. Les flèches à la surface du cGld indiquent les limites dorsale et ventrale du noyau. VB, ventrobasal; cGld, corps genouillé latéral dorsal. B. Marquage antérograde de la projection rétine-Cl transitoire chez un hamster âgé de 3 jours, après injection de HRP dans l'œil contralatéral. Coupe parasagittale, face dorsale en haut, région caudale à droite. Les faisceaux d'axones rétinofuges atteignent quasiment l'extrémité caudale du $\mathrm{Cl}$ (flèches noires épaisses). ${ }^{*}$ : aqueduc de Sylvius ; $\mathrm{Cl}$ : colliculus inférieur ; CRV : cervelet. HRP révélée par la tétraméthylbenzidine (TMB) sur des coupes de $40 \mu m$ d'épaisseur, contrecolorées au rouge neutre. Même échelle pour les deux coupes.

thalamus, adjacent au noyau visuel, vers une structure mésencéphalique auditive - le colliculus inférieur et enfin vers le pont et la substance noire. La projection ectopique de la rétine au thalamus somatosensoriel est développée pendant les trois premiers jours postnataux, puis disparaît normalement le quatrième. La projection rétinienne ectopique au colliculus inférieur atteint son maximum aux troisième et quatrième jours postnataux pour disparaître au cours de la seconde semaine post-natale [31] (figure 4).

L'“ exubérance " qui conduit au développement de projections rétinofuges vers des cibles ectopiques peut se manifester à des stades différents de la croissance des axones rétiniens, stades de croissance morphologiquement différenciables [32, 33] (figure 5 , p. 852) : dans une première phase d'élongation, les axones poussent dans le tractus optique vers le mésencéphale sans émettre de collatérales ; puis ils envoient des collatérales peu ou pas branchées dans des cibles sous-corticales di- et mésencéphaliques, visuelles ou non; enfin survient l'arborisation des terminaisons $\mathrm{m} / \mathrm{s} n^{\circ} 9$, ool. 6 , nooembre 90 dans les cibles. Si la projection de la rétine vers le mésencéphale auditif résulte d'une exubérance d'élongation des axones rétiniens qui dépassent la limite postérieure du colliculus supérieur, la projection de la rétine vers le thalamus somatosensoriel résulte d'une exubérance de la phase de collatéralisation. Cette dernière est formée par des collatérales de fibres du tractus optique profond, fibres qui chez l'adulte, traversent le diencéphale sans s'y projeter.

Au cours du développement normal, il est classique que les neurones se projetant initialement vers plusieurs cibles éliminent certaines collatérales et en maintiennent d'autres. Ce processus permet, dans une population homogène de neurones immatures, l'individualisation de plusieurs populations cellulaires, par le biais d'une élimination différentielle de collatérales. Ainsi chez le hamster, parmi les fibres rétiniennes qui envoient une collatérale au diencéphale, celles qui traversent le corps genouillé latéral en profondeur élimineront cette collatérale pour ne maintenir que leur projection au mésencéphale alors que celles qui passent à la surface main- tiendront les deux projections, di- et mésencéphaliques. Ce mode de développement, où les neurones sélectionnent leurs cibles à partir d'un répertoire initial plus large, peut s'interpréter, entre autres, comme une stratégie qui économise le coût génétique du développement, ou comme un réservoir de variabilité précieux sur le plan évolutif [12]. Au niveau cortical, ce mécanisme pourrait rendre compte de l'émergence de différences régionales au cours du développement $[34,35]$.

\section{Stabilisation et induction de projections visuelles ectopiques chez le hamster Mécanismes cellulaires impliqués dans les phénomènes de stabilisation/élimination}

Chez les hamsters opérés à la naissance, les axones rétiniens dont l'environnement a été fortement perturbé sélectionnent de nouvelles cibles. La projection rétinienne au thalamus somatosensoriel, observée chez les animaux adultes opérés à la naissance, semble bien résulter de la stabilisation d'une projection transi- 

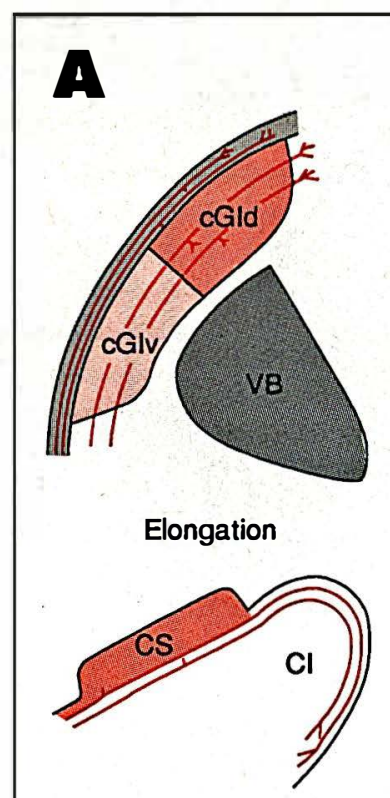

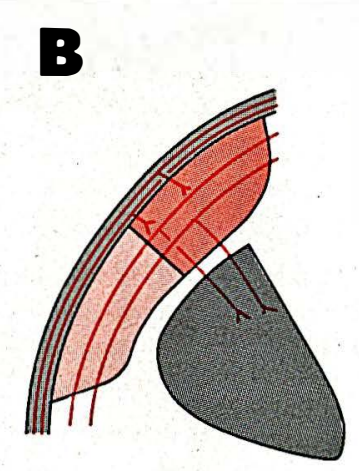

Collatéralisation

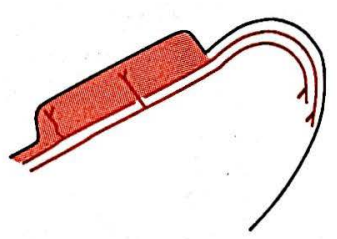

\section{C}

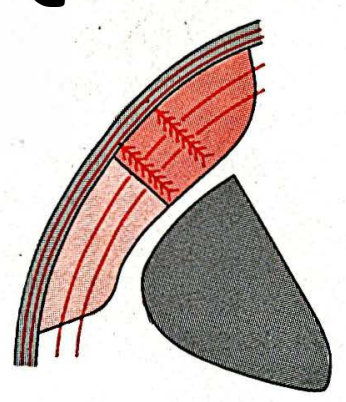

Arborisation

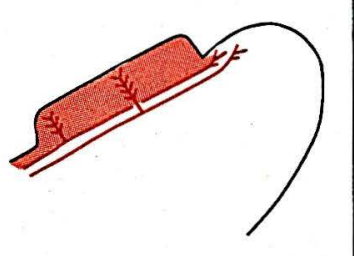

Figure 5. Représentation très schématique des trois stades de croissance des axones rétiniens. Ligne du haut: sections frontales du diencéphale. $c G(d(v)$, corps genouillé latéral dorsal (ventral); VB, noyau ventrobasal. Ligne du bas : sections parasagittales du mésencéphale. CS(I) colliculus supérieur (inférieur). A : élongation des axones dans le tractus optique. Ils ne sont pas branchés; certains dépassent le colliculus supérieur pour atteindre le colliculus inférieur. $B$ : collatéralisation : les axones rétiniens envoient des branches peu ou pas ramifiées vers leurs cibles. $C$ : arborisation des terminaisons rétiniennes dans leurs cibles. toire établie par le tractus optique profond et de l'induction du développement de nouvelles collatérales à superficiel [36]. Dans le cas de la projection au thalamus auditif, à aucun stade (embryonnaire ou périnatal) du développement normal du hamster, on n'observe de projection transitoire de la rétine sur cette structure. Son établissement semble dû à une repousse des axones rétiniens déclenchée par la chirurgie néonatale, après un délai de 48 heures maximum [37].

Il est probable que les mêmes facteurs contrôlent, chez les animaux opérés, la stabilisation des connexions transitoires et, chez les animaux normaux, les mécanismes de stabilisation/élimination des projections immatures. Notre modèle expérimental illustre bien l'action du milieu environnant dans le contrôle du maintien ou de l'élimination de propartir des fibres du tractus optique ple remarquable est fourni par certains neurones du cortex occipital du rat. Ces neurones projettent transitoirement à trois cibles au cours de leur développement précoce, le mésencéphale, le pont et la moelle épinière, puis ils éliminent sélectivement leur collatérale spinale pour ne maintenir que la collatérale au mésencéphale ou au pont [7]. Si ces neurones occipitaux sont greffés dans le cortex pariétal à la naissance, ils maintiennent leur projection à la moelle épinière. Réciproquement, des neurones pariétaux transplantés dans le cortex occipital éliminent la projection spinale qu'ils développent et maintiennent normalement [35].

Le rôle de signaux extrinsèques aux neurones dans ces phénomènes est maintenant bien établi : au niveau des cibles, les fibres nerveuses sont en compétition pour l'espace synaptique terminal ainsi que pour des substances trophiques, disponibles en quantité limitée dans les cibles [38].
Par ailleurs, l'activité électrique des cellules nerveuses joue un rôle fondamental dans l'élimination ou dans le renforcement et la stabilisation fonctionnelle de certains contacts synaptiques. Ainsi l'activité spontanée, vraisemblablement corrélée dans les cellules ganglionnaires voisines, est un élément organisateur fondamental de la voie rétino-géniculocorticale, comme le montrent les expériences d'injection intraoculaire de TTX $[39,40]$. Dans le processus de maturation du système nerveux central interviennent également des mécanismes dépendants de l'activité évoquée, en particulier de la corrélation entre l'activité pré- et postsynaptique $[41,42]$.

L'observation en microscopie électronique, chez le hamster nouveau-né, de synapses immatures entre des axones rétiniens et des neurones du thalamus somatosensoriel et du colliculus inférieur [43], conforte l'idée qu'un processus fonctionnel lié à l'activité synaptique intervient dans le maintien ou l'élimination de la projection de la rétine vers le thalamus somatosensoriel. Des synapses fonctionnelles, établies par des projections rétiniennes destinées à disparaître dans la suite du développement sur les cellules relais du corps genouillé latéral, ont été trouvées chez le chat nouveau-né [44].

D'autres facteurs tels que la tendance des neurones à maintenir une certaine quantité de contacts synaptiques et d'arborisations terminales [20], ou la proximité géographique entre un axone en croissance et un site de terminaison potentiel, c'est-à-dire vacant [37], peuvent aussi intervenir.

\section{Conclusion}

Le remodelage qui affecte le SNC au cours de son développement normal est contrôlé par des facteurs génétiques, mais aussi par des facteurs épigénétiques dont le rôle semble d'autant plus important que l'on s'adresse à des espèces plus élevées dans l'échelle de l'évolution. Il est maintenant bien établi que l'expérience sensorielle précoce contrôle, pour une part importante, le développement postnatal du SNC; le déficit fonctionnel d'un organe des sens peut entraîner des altérations 
centrales - morphologiques et fonctionnelles - considérables [45]. A l'inverse, la plasticité du SNC et les mécanismes compensatoires qu'elle autorise, peuvent servir de base à la récupération de certains déficits périnataux liés à des lésions nerveuses centrales ou périphériques. Il est généralement admis que les réponses du SNC aux lésions précoces conduisent à des récupérations fonctionnelles plus importantes que les réponses à des lésions plus tardives. Les études menées par G.E. Schneider chez le hamster [46] ont mis en évidence que les réorganisations fonctionnelles et anatomiques induites par une lésion néonatale du cortex visuel ou du colliculus supérieur, sont généralement bien adaptées. Les études menées chez le rat donnent des résultats moins clairs. Les performances visuelles des animaux dont le cortex visuel a été détruit à la naissance ne sont pas meilleures que celles des animaux dont le cortex visuel a été détruit à l'âge adulte (pour revue [9]). Il semble néanmoins que, plus la lésion d'une structure impliquée dans une fonction donnée intervient tôt dans le développement, plus cette fonction est épargnée, mais plus les performances cognitives globales, toujours difficiles à quantifier, sont atteintes $[9,11]$. Cette observation a également été faite chez l'homme [47]. Il reste que l'on peut espérer compenser partiellement des déficits neurologiques détectés précocement grâce à la mise en jeu "forcée " de structures capables de supplanter les structures déficientes.

Enfin, nos résultats expérimentaux permettent de spéculer sur la possibilité de réparer chirurgicalement un cerveau adulte lésé, dans le futur. Dans la mesure où il est possible d'induire, dans le cerveau adulte, la repousse de fibres dans des " ponts " formés par des bouts de nerfs périphériques [22], et dans la mesure où la compréhension des mécanismes cellulaires impliqués dans l'inhibition de la croissance des fibres nerveuses dans le cerveau adulte a beaucoup progressé [21], peut-être est-il possible d'imaginer faire repousser, dans des cerveaux adultes lésés, de nouvelles voies capables d'établir des circuits fonctionnels dans les régions intactes

\section{Summary}

Reorganisation of visual pathways following early brain lesions

Early brain lesions are followed by important morphological and functional reorganisations. When the principal targets of retinofugal axons are ablated in newborn hamsters and an alternative terminal space is created by partial deafferentation of the somatosensory or auditory thalamic nuclei, optic axons are induced to make permanent projections in these nuclei. The experimentally induced abnormal visual projections display a topographic arrangement in their ectopic targets. The synapses, that retinal fibers establish with relay cells in the somatosensory and auditory thalamic nuclei, exhibit morphological features that are similar to those of normal somatosensory and auditory projections. Electrophysiological studies performed in the somatosensory cortex of hamsters with a retina-somatosensory thalamus projection have shown this projection to be functional. They are at least two different mechanisms by which such a reorganization of the visual projection can occur; one is the capacity for neurons to modify the elimination of supernumerary transitory connections, the other is the sprouting of new fibers. The possibility for these new pathways to compensate for the perceptive deficits due to the lesions is now under investigation. The experiments reveal the mechanisms of normal and pathologic development and raise the possibility of surgical repair of the mature brain.

\section{TIRÉS A PART}

C. Métin. 